Jap. J. M. Sc. \& Biol., 12, 473-478, 1959

\title{
ANTI-CANCER EFFECTS OF NAPHTHOQUINONE DERIVATIVES TESTED BY A SCREENING WITH EHRLICH ASCITES CELLS IN MICE*
}

In the previous study (Egashira et $a l$., 1959), the procedure and evaluation of results of an anti-cancer screening system, using ascitic and solid forms of the Ehrlich tumor cells, were standardized on the basis of observations on inoculated animals analyzed from several viewpoints.

Among various kinds of synthetic chemical compounds which had been tested through this screening system, naphthoquinone derivatives were selected to be described herein. There have been few reports concerning the anti-cancer effects of naphthoquinones. Miyaki et al. (1956) synthetized several arylthionaphthoquinones and examined them by a cytological method. Abe et al. (1959) reported the result of a cell agar plate screening of the above compounds. In the present report, the screening result of 44 new naphthoquinone derivatives including the above will be described.

Naphthoquinone derivatives tested were divided depending on their chemical structures into 4 groups: A) alkylthionaphthoquinones (9 compounds), B) arylthionaphthoquinones (17), C) halogenonaphthoquinones (12), and D) miscellaneous (6).

The standards for procedure and evaluation of results were as described and tabulated in the previous paper (Egashira et al., 1959). If a drug showed any effect either in intraperitoneal (i.p.) or subcutaneous (s.c.) group, the test was repeated so far as the amount of drug was permitted. When one proved to be toxic in the standard dose of $1200 \mu \mathrm{g}$, a smaller amount was tried repeatedly.

The results of screening of 44 naphthoquinones are summarized in Tables 1-4. Three compounds in group A (Table 1), 2-methylthio-, 2,3-dimethylthio-, and 2-methylthio3-Br-naphthoquinones, were determined to be effective to some extent both in i.p. and s.c.; 7 in group $\mathrm{B}$ (Table 2), especially 2-(p-toluyl)-thionaphthoquinone, and 5 in group $\mathrm{C}$ (Table 3), especially 2-Cl- and 2,3-Cl-types showed effectiveness only in i.p., concerning the increase in longevity and viability.

According to the result of primary screening stated above, some effective drugs were examined again, the administration being started 3 days, instead of 1 day, after tumor inoculation (the secondary screening). Some of them were proved still effective through the procedure. The result on 2,3-dimethylthionaphthoquinone is illustrated in Fig. 1.

In summary, totally 44 naphthoquinone derivatives were examined throuph a standardized screening system. Effective compounds belonged to thio-type and some relationship of the function to the chemical structure was suggested.

\section{REFERENCES}

Miyaki, K., Ugami, S., Ikeda, N. \& Kuretani, K. (1956) : The tumor-inhibiting activities of arylnaphthoquinones. Pharm. Bull., 4, 417-419.

Abe, M., Mryaki, K., Mizuno, D., Narita, N., Takeuchi, T., Ukita, T. \& Yamamoto, T. (1959): The cell agar plate screening for antitumor cell effect of 1300 organic compounds.

* Aided in part by the fund of the Education Ministry. 

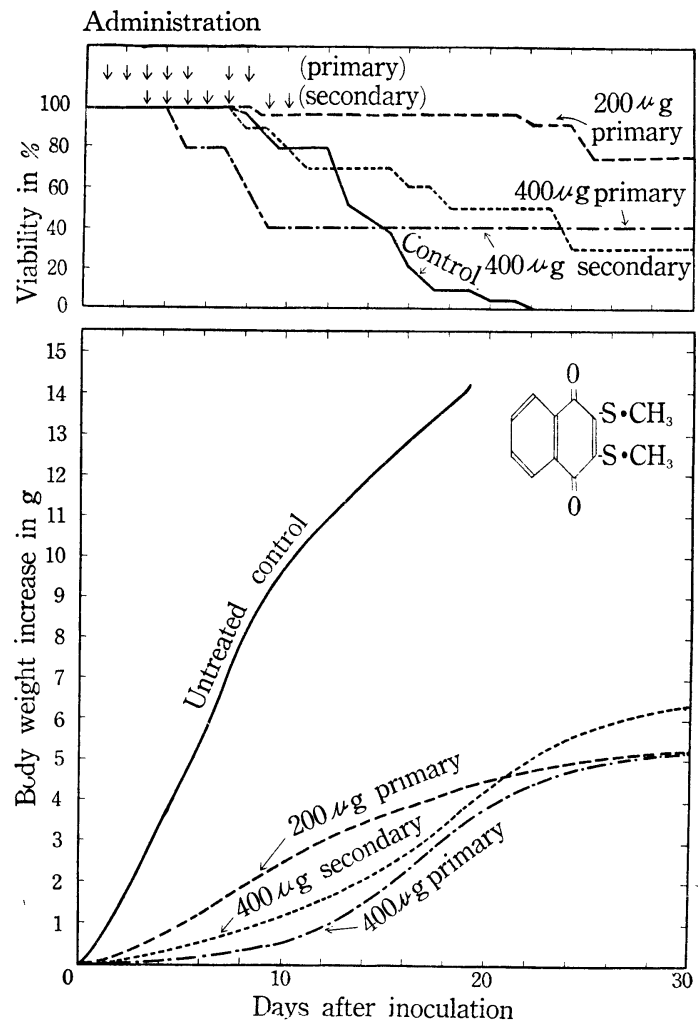

Fig. 1. Results of 2,3-dimethylthionaphthoquinone administration into mice receiving i.p. $2 \times 10^{6}$ Ehrlich tumor cells. Administration started 24 hours and 3 days after inoculation in primary and secondary screening procedures, respectively.

Jap. J. M. Sc. \& Biol., 12, 175-292.

Egashira, Y., Takano, K., Yamada, M., Hirokawa, Y., Mizuno, D., Abe, M. \& Masamune, Y. (1959): Standardization of procedures for cancer chemotherapy screening with Ehrlich ascites tumor cells. Jap. J. M. Sc. \& Biol., 12, 463-470.

Department of Pathology, National

Institute of Heath, Tokyo

Department of Chemistry, National

Institute of Health, Tokyo

Faculty of Pharmacy, Chiba University, Chiba

Received: December 30th, 1959
KOUICHI TAKANO

MASA-ATSU YAMADA

YASUKO HIROKAWA

DEN'ICHI MIZUNO

MrHoko ABE

KOMEI MIYAKI

NISABURO IKEDA

高野宏一・山田正篤・広川康子（国立予防衛生研究所病理部）

水野伝一・阿部美穂子（同化学部）

宮木高明・池田仁三郎 (千葉大学楽学部) 
Table 1. Screening results on alkylthionaphthoquinones

\begin{tabular}{|c|c|c|c|c|c|}
\hline \multirow{3}{*}{ Drug } & \multirow{3}{*}{$\begin{array}{l}\text { Dose } \\
\text { (per mouse } \\
\text { per day) }\end{array}$} & \multirow{3}{*}{ Toxicity } & \multicolumn{3}{|c|}{ Tumor type } \\
\hline & & & \multicolumn{2}{|c|}{ Ascites } & \multirow{2}{*}{ 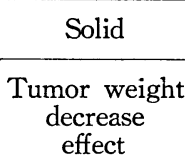 } \\
\hline & & & Viability & $\begin{array}{l}\text { Longevity } \\
\text { increase } \\
\text { effect }\end{array}$ & \\
\hline $\mathrm{R}_{2}:-\mathrm{S} \cdot \mathrm{CH}_{3}$ & $\begin{array}{l}1200^{\mu g} \\
200 \\
200\end{array}$ & $\begin{array}{c}(1) \\
10 / 10 \\
0 / 10 \\
0 / 5\end{array}$ & $\begin{array}{r}(2) \\
4 / 5\end{array}$ & $\begin{array}{l}\text { (3) } \\
+\end{array}$ & $\begin{array}{l}(4) \\
\pm \\
\pm\end{array}$ \\
\hline$-\mathrm{S} \cdot \mathrm{C}_{2} \mathrm{H}_{5}$ & $\begin{array}{r}1200 \\
600 \\
400 \\
200\end{array}$ & $\begin{array}{l}10 / 10 \\
2 / 5 \\
0 / 5 \\
0 / 5\end{array}$ & & & $\begin{array}{l}+ \\
\pm \\
-\end{array}$ \\
\hline$-\mathrm{S}_{3} \cdot \mathrm{C}_{3} \mathrm{H}_{7}$ & $\begin{array}{r}1200 \\
200\end{array}$ & $\begin{array}{r}10 / 10 \\
0 / 10\end{array}$ & $0 / 5$ & - & - \\
\hline$-\mathrm{S} \cdot \mathrm{CH}_{2} \mathrm{COOH}$ & $\begin{array}{r}12 \mathrm{CO} \\
200\end{array}$ & $\begin{array}{c}10 / 10 \\
0 / 5\end{array}$ & & & - \\
\hline $\begin{array}{l}\mathrm{R}_{2}:-\mathrm{S} \cdot \mathrm{CH}_{3} \\
\mathrm{R}_{3}:-\mathrm{S} \cdot \mathrm{CH}_{3}\end{array}$ & $\begin{array}{r}1200 \\
400 \\
200 \\
200 \\
200\end{array}$ & $\begin{array}{l}7 / 10 \\
5 / 10 \\
1 / 10 \\
0 / 20 \\
0 / 20\end{array}$ & $\begin{array}{l}1 / 1 \\
2 / 2 \\
4 / 10 \\
9 / 10\end{array}$ & $\begin{array}{l}H \\
H \\
+ \\
H\end{array}$ & $\begin{array}{l}+ \\
\pm \\
+ \\
\pm \\
\pm\end{array}$ \\
\hline $\begin{array}{l}-\mathrm{S} \cdot \mathrm{CH}_{3} \\
-\mathrm{Cl}\end{array}$ & $\begin{array}{r}1200 \\
600 \\
200\end{array}$ & $\begin{array}{l}10 / 10 \\
5 / 5 \\
0 / 5\end{array}$ & & & - \\
\hline $\begin{array}{l}-\mathrm{S} \cdot \mathrm{CH}_{3} \\
-\mathrm{Br}\end{array}$ & $\begin{array}{r}1200 \\
200\end{array}$ & $\begin{array}{l}5 / 5 \\
1 / 10\end{array}$ & $3 / 4$ & H & + \\
\hline$-\mathrm{S} \cdot \mathrm{CH}_{3}$ & 1200 & $0 / 10$ & $0 / 5$ & \pm & - \\
\hline$-\mathrm{NH}-<$ & 800 & $0 / 5$ & & & - \\
\hline $\begin{array}{l}-\mathrm{S} \cdot \mathrm{CH}_{3} \\
-\mathrm{NH} \cdot \mathrm{CH}_{2}-\end{array}$ & 1200 & $0 / 10$ & $0 / 5$ & - & - \\
\hline
\end{tabular}

(1) No. of deaths within 9 days/No. of mice used.

(2) No. of survivals after 30 days/that over 9 days.

(3) Evaluated by increase ratio of mean longevity to control.

(4) Evaluated by ratio of mean tumor weight to control. 
Table 2. Screening results on arylthionaphthoquinones

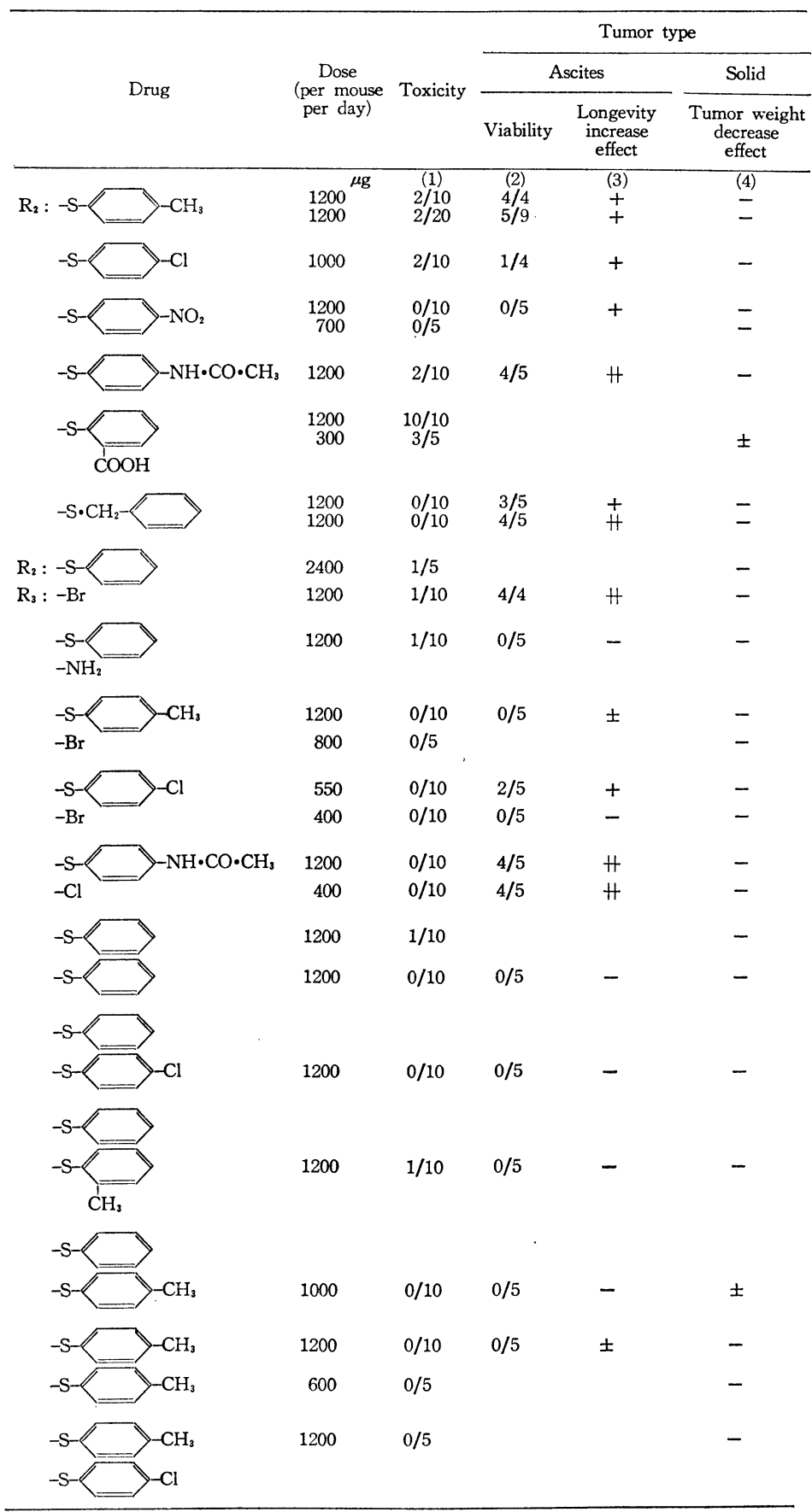

Cf. footnotes of Table 1. 
Table 3. Screening results on halogenonaphthoquinones

\begin{tabular}{|c|c|c|c|c|c|}
\hline \multirow{3}{*}{ Drugs } & \multirow{3}{*}{$\begin{array}{c}\text { Dose } \\
\text { (per mouse } \\
\text { per day) }\end{array}$} & \multirow{3}{*}{ Toxicity } & \multicolumn{3}{|c|}{ Tumor type } \\
\hline & & & \multicolumn{2}{|c|}{ Ascites } & \multirow{2}{*}{ 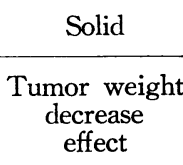 } \\
\hline & & & Viability & $\begin{array}{l}\text { Longevity } \\
\text { increase } \\
\text { effect }\end{array}$ & \\
\hline $\mathrm{R}_{2}:-\mathrm{Cl}$ & $\begin{array}{c}1200^{\mu \mathrm{g}} \\
300 \\
100\end{array}$ & $\begin{array}{l}(1) \\
10 / 10 \\
10 / 10 \\
0 / 10\end{array}$ & $\begin{array}{l}(2) \\
4 / 5\end{array}$ & (3) & (4) \\
\hline$-\mathrm{Br}$ & $\begin{array}{r}1200 \\
300\end{array}$ & $\begin{array}{l}10 / 10 \\
2 / 5\end{array}$ & & & + \\
\hline $\begin{array}{l}\mathrm{R}_{2}:-\mathrm{Cl} \\
\mathrm{R}_{3}:-\mathrm{Cl} \\
\mathrm{R}_{5}:-\mathrm{NH}_{2}\end{array}$ & $\begin{array}{r}1200 \\
600 \\
200\end{array}$ & $\begin{array}{r}10 / 10 \\
10 / 10 \\
2 / 10\end{array}$ & $4 / 5$ & $H$ & - \\
\hline $\begin{array}{l}-\mathrm{Cl} \\
-\mathrm{Cl} \\
-\mathrm{NO}_{2}\end{array}$ & 1200 & $7 / 10$ & $2 / 3$ & H & - \\
\hline $\begin{array}{l}\mathrm{R}_{2}:-\mathrm{Br} \\
\mathrm{R}_{3}:-\mathrm{Br}\end{array}$ & 1200 & $4 / 10$ & $2 / 3$ & + & - \\
\hline $\begin{array}{l}-\mathrm{Cl} \\
-\mathrm{NH}_{2}\end{array}$ & 1200 & $0 / 10$ & $0 / 5$ & - & \pm \\
\hline $\begin{array}{l}-\mathrm{Br} \\
-\mathrm{NH} \cdot \mathrm{CO} \cdot \mathrm{CH}_{3}\end{array}$ & $\begin{array}{r}1200 \\
400\end{array}$ & $\begin{array}{l}5 / 5 \\
3 / 10\end{array}$ & $3 / 4$ & + & - \\
\hline $\begin{array}{l}-\mathrm{Cl} \\
-\mathrm{N}\left(\mathrm{CH}_{3}\right)_{2}\end{array}$ & 1200 & $4 / 10$ & $1 / 1$ & $H$ & - \\
\hline$-\mathrm{- \textrm {NH }} \cdot \mathrm{CH}\left\langle{ }_{\mathrm{C}_{2} \mathrm{H}_{5}}^{\mathrm{CH}_{3}}\right.$ & 1200 & $0 / 10$ & $0 / 5$ & - & - \\
\hline $\begin{array}{l}-\mathrm{Cl} \\
-\mathrm{NH} \cdot\left(\mathrm{CH}_{2}\right)_{2} \mathrm{COOH}\end{array}$ & 1200 & $0 / 10$ & $0 / 5$ & - & - \\
\hline $\begin{array}{l}-\mathrm{Cl} \\
-\mathrm{NH}\left(\mathrm{CH}_{2}\right)_{3}\end{array}$ & 1000 & $1 / 10$ & $0 / 5$ & - & - \\
\hline $\begin{array}{l}-\mathrm{Cl} \\
-\mathrm{NH}-\end{array}$ & 1200 & $0 / 10$ & $0 / 5$ & - & - \\
\hline
\end{tabular}

Cf. footnotes of Table 1. 
Table 4. Screening results on miscelleneous naphthoquinones

\begin{tabular}{|c|c|c|c|c|c|}
\hline \multirow{3}{*}{ Drug } & \multirow{3}{*}{$\begin{array}{c}\text { Dose } \\
\text { (per mouse } \\
\text { per day) }\end{array}$} & \multirow{3}{*}{ Toxicity } & \multicolumn{3}{|c|}{ Tumor type } \\
\hline & & & \multicolumn{2}{|c|}{ Ascites } & \multirow{2}{*}{$\frac{\text { Solid }}{\begin{array}{c}\text { Tumor weight } \\
\text { decrease } \\
\text { effect }\end{array}}$} \\
\hline & & & Viability & $\begin{array}{l}\text { Longevity } \\
\text { increase } \\
\text { effect }\end{array}$ & \\
\hline $\mathrm{R}_{2}:-\mathrm{NH}_{2}$ & $1200^{\mu \mathrm{g}}$ & $\begin{array}{c}(1) \\
0 / 10\end{array}$ & $\begin{array}{l}(2) \\
1 / 5\end{array}$ & $\begin{array}{l}(3) \\
\pm\end{array}$ & $\stackrel{(4)}{-}$ \\
\hline$-\mathrm{NH}$ & $\begin{array}{l}1200 \\
1200\end{array}$ & $\begin{array}{l}0 / 10 \\
0 / 5\end{array}$ & $0 / 5$ & - & \pm \\
\hline$-\mathrm{NH}-<$ & 1200 & $0 / 10$ & $0 / 5$ & - & - \\
\hline$-\mathrm{NH}-\mathrm{C}$ & 1200 & $0 / 5$ & $1 / 5$ & - & \\
\hline $\mathrm{NH} \cdot \mathrm{CH}_{2}$ & 1200 & $0 / 10$ & $0 / 5$ & - & - \\
\hline \multicolumn{6}{|l|}{$\mathrm{R}_{2}:-\mathrm{NO}_{2}$} \\
\hline $\mathrm{R}_{3}:-\mathrm{OH}$ & 1200 & $0 / 10$ & $0 / 5$ & - & - \\
\hline
\end{tabular}

Cf. footnotes of Table 1. 